\section{Fermilab director resigns over funding}

IN an effort to stabilise the funding of high energy physics (HEP), the US Department of Energy has decided to recommend that the HEP budget should be kept at a constant value of $\$ 300$ million (in 1979 prices) over the next few years.

According to the department, this sum-which breaks down into $\$ 200$ million for operating costs, $\$ 40$ million for equipment, and $\$ 60$ million for construction-will guarantee an active HEP programme based on three national centres, the Brookhaven $\mathrm{Na}$ tional Laboratory on Long Island, the Fermi National Accelerator Laboratory in Illinois, and the Stanford Linear Accelerator Center (SLAC) in California.

However, although the department's proposed commitment is considerably higher than two years ago, when the HEP budget dropped to about $\$ 240$ million after reaching a peak of about \$500 million (both in 1979 prices) in 1970 , it is still less than many scientists would like. And in protest at the department's decision not to speed up the construction of the energy doubler at Fermilab, Dr Robert Wilson, director of the laboratory, has carried out a threat made two months ago and submitted his resignation.

The Department of Energy's decision to keep HEP funding constant at a level slightly higher than the $\$ 290$ million proposed for fiscal year 1979 was taken after long and detailed discussion with the high energy physics community, in particular over the construction of the $400 \mathrm{GeV}$ proton-proton colliding beam facility (ISABELLE) at Brookhaven which the department has proposed should start in 1979.

"Before agreeing to support ISABELLE, I asked what would make sense in terms of a long-term high energy physics programme?", Dr John Deutch, director of the department's Office of Energy Research, said last week. "I was not prepared to support the construction of a major new facility unless it was in the context of a balanced and aggressive high energy physics programme." Dr Deutch said that the decision to maintain a constant budget in real terms over the next few years was consistent with the consensus of the high energy physics community over the type of support that was required, and that it would provide some stability for future planning. A similar scheme was also being developed for funding nuclear physics.

The decision was welcomed by $\mathrm{Dr}$ Sidney Drell, deputy director and chairman of the department's High Energy Physics Advisory Panel (HEPAP), who pointed out that ever since the Ramsey report of the early 1960s. 1960s, physicists have been asking for a stable basis for long-range planning. "Stable funding expectations will be very useful to us in terms of allocating resources, particularly in view of the fact that major HEP instruments can take seven years or so to build", he said. He added that the general level of funding was only "the minimum viable level" compatible with a three-centre programme, and there would still be many opportunities lost for good physics. According to Dr Drell, the proposed funding levels represent a $25 \%$ drop in operating funds for HEP facilities from their peak at the end of the

Dr Wilson's decision to resign as director of Fermilab is a result of his failure to persuade the Department of Energy to bring forward the construction of the energy doubler, which by adding a second ring of superconducting magnets will eventually enable Fermilab both to save power costs and to reach energies of $1,000 \mathrm{GeV}$ or more. Although the department is proposing to allocate $\$ 10$ million towards construction of the doubler in the fiscal year 1979, Dr Wilson had asked for
\$35 million, which would have been sufficient to complete the project during the year.

In his letter of resignation to the Universities Research Association, which runs Fermilab, Dr Wilson said that current restrictions of funding meant that the laboratory was operating at about half its potential capacity, a predicament that was particularly serious in view of the relatively high funding enjoyed by the European Centre for Nuclear Research (CERN) in Geneva, which recently brought its own $400 \mathrm{GeV}$ accelerator into successful operation.

"Our scheme to leapfrog CERN's financial advantage by increasing the Fermilab proton energy to $1,000 \mathrm{GeV}$ through the application of superconductivity has been confounded by indecisive and subminimal support," Dr Wilson said. He added that he had felt he was unable to continue to give the impression that he could responsibly direct Fermilab without a substantial increase in funding.

Although Dr Wilson is resigning as director, he has said that he intends to remain at Fermilab to work on the energy doubler project.

David Dickson

\title{
Salyut-6 used for weightlessness experiments
}

THE long sojourn of cosmonauts Yurii Romanenko and Georgii Grechko aboard Salyut- 6 represents far more than an attempt to recapture the space endurance record. Soviet space planning has always been based on the idea of permanent orbital stations, indeed, Tsiolkovskii, "the father of Soviet cosmonautics" envisaged such stations as a sine qua non to manned lunar or interplanetary flight. The rumours which followed the Soyuz-11 disaster, which attributed the cosmonaut's deaths to the effect of prolonged weightlessness, were traumatic to space planners and amateur enthusiasts alike, and although subsequent investigations proved that the tragedy was in fact caused by a defective seal and consequent depressurisation, research into the physiology of weightlessness is a major concern to the Soviet manned space programme.

Unfortunately, weightlessness research is something which must largely be carried out in situ, and there is little scope for ground-level simulation. A 'hydro-weightlessness' tank is used to train cosmonauts for the difficult mechanical operations of station maintenance. Poland, as part of its contribution to the Interkosmos project, is said to be working on the biomedical effects of weightlessness, and an interesting experiment was recently

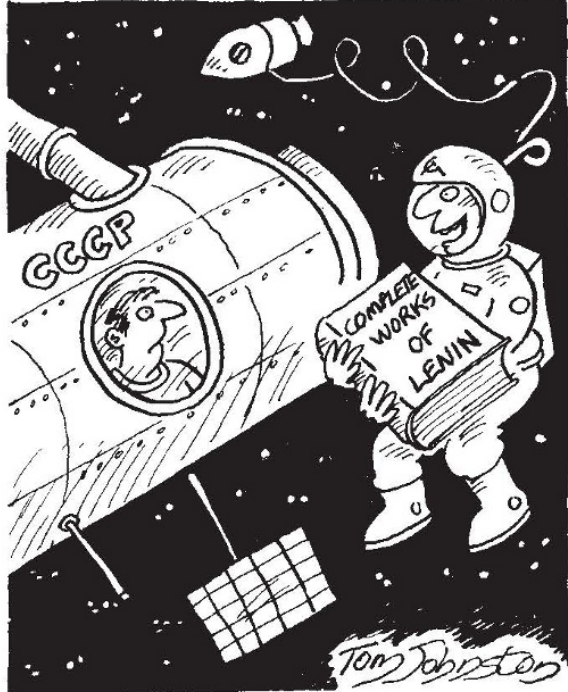

'You did say any kind of moral support, comrade!'

completed in Leningrad where for six months 18 volunteers lay "in a very inconvenient posture, with their feet several degrees higher than their heads", able to wash, shave, eat, and read, but "only allowed to turn from side to side, without rising even a little".

That experiment, which was presumably intended to simulate spaceflight demineralisation, proved, according to its scientific director, Professor Leonid Kakurin, to be a great success. 